\title{
Carbonized Polymer Dots with Tunable Room-Temperature Phosphorescence Lifetime and Wavelength
}

Chunlei Xia ${ }^{\dagger}$, Shoujun Zhut, ${ }^{\dagger}$, Shi-Tong Zhang ${ }^{\dagger}$, Qingsen Zeng ${ }^{\dagger}$, Songyuan Tao ${ }^{\dagger}$, Xuzhou Tian $^{\dagger}$, Yunfeng Lit, and Bai Yang *广

${ }^{\dagger}$ State Key Laboratory of Supramolecular Structure and Materials, College of Chemistry, Jilin University, Changchun, 130012, P. R. China.

Key Laboratory of Organ Regeneration and Transplantation of the Ministry of Education, The First Hospital of Jilin University, Changchun, 130061, P. R. China.

*E-mail: byangchem@jlu.edu.cn

\section{Table of Contents}

1. Figure S1. The prepared AN-CPDs powder under daylight and $365 \mathrm{~nm}$ UV lamp.

2. Figure S2. The display of the gram-scale AN-CPDs obtained by scale up production.

3. Figure S3. The fitting results of RTP decay. Phosphorescence decay spectra and fitting curves of AN-CPDs with different carbonization degree.

4. Figure S4. FT-IR spectra of the AN-CPDs with different carbonization degree.

5. Figure S5-S10. The optical properties of AN-CPDs with different carbonization degree.

6. Figure S11. Scheme of possible structures formed during the hydrothermal treament process. 
7. Figure S12. The optimized conformation of the predicted cyclic imide structure.

8. Figure S13. N 1s and O 1s high-resolution XPS spectra of the AN-CPDs-150, AN-CPDs, AN-CPDs-250 and AN-CPDs-300.

9. Figure S14. The photo display of AN-CPDs as the encrypted security ink.

10. Figure S15. RTP emission performance contrast after 200 days to show the stability.

11. Table S1. The RTP lifetime fitting results of the AN-CPDs with different carbonization degree and CPDs-contrast.

12. Table S2. The theoretical calculations results using the delta-SCF method.

13. Table S3. Relative contents of different functional groups in the AN-CPDs-150, AN-CPDs, AN-CPDs-250, AN-CPDs-300, based on the analysis of C1s high-resolution XPS spectra. 


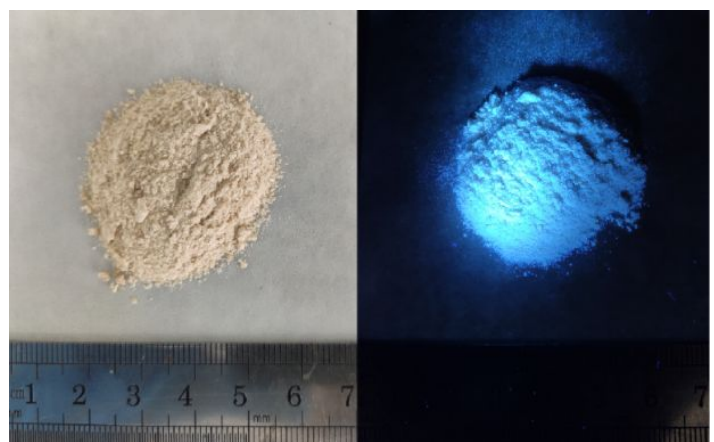

Figure S1. The prepared AN-CPDs powder under daylight and $365 \mathrm{~nm}$ UV lamp.

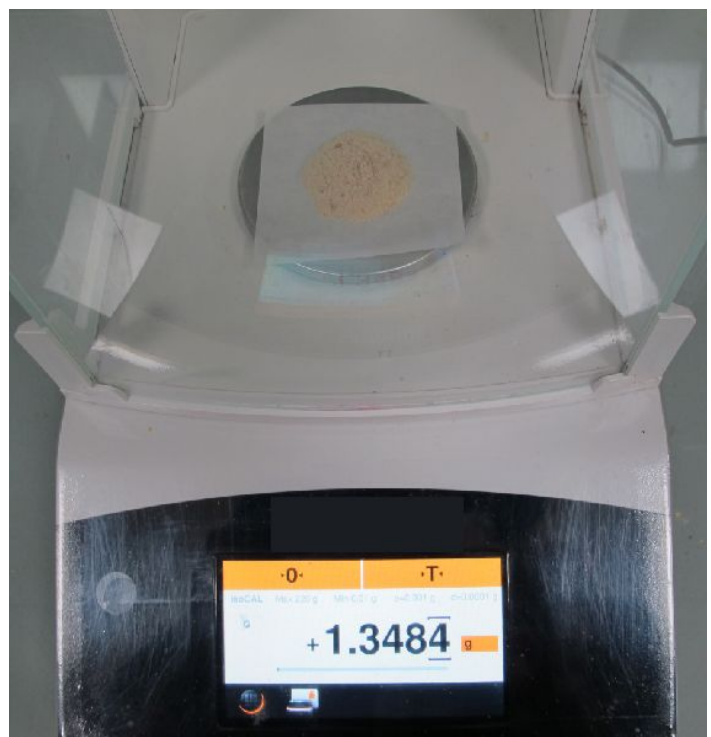

Figure S2. The display of the gram-scale AN-CPDs obtained by scale up production. 

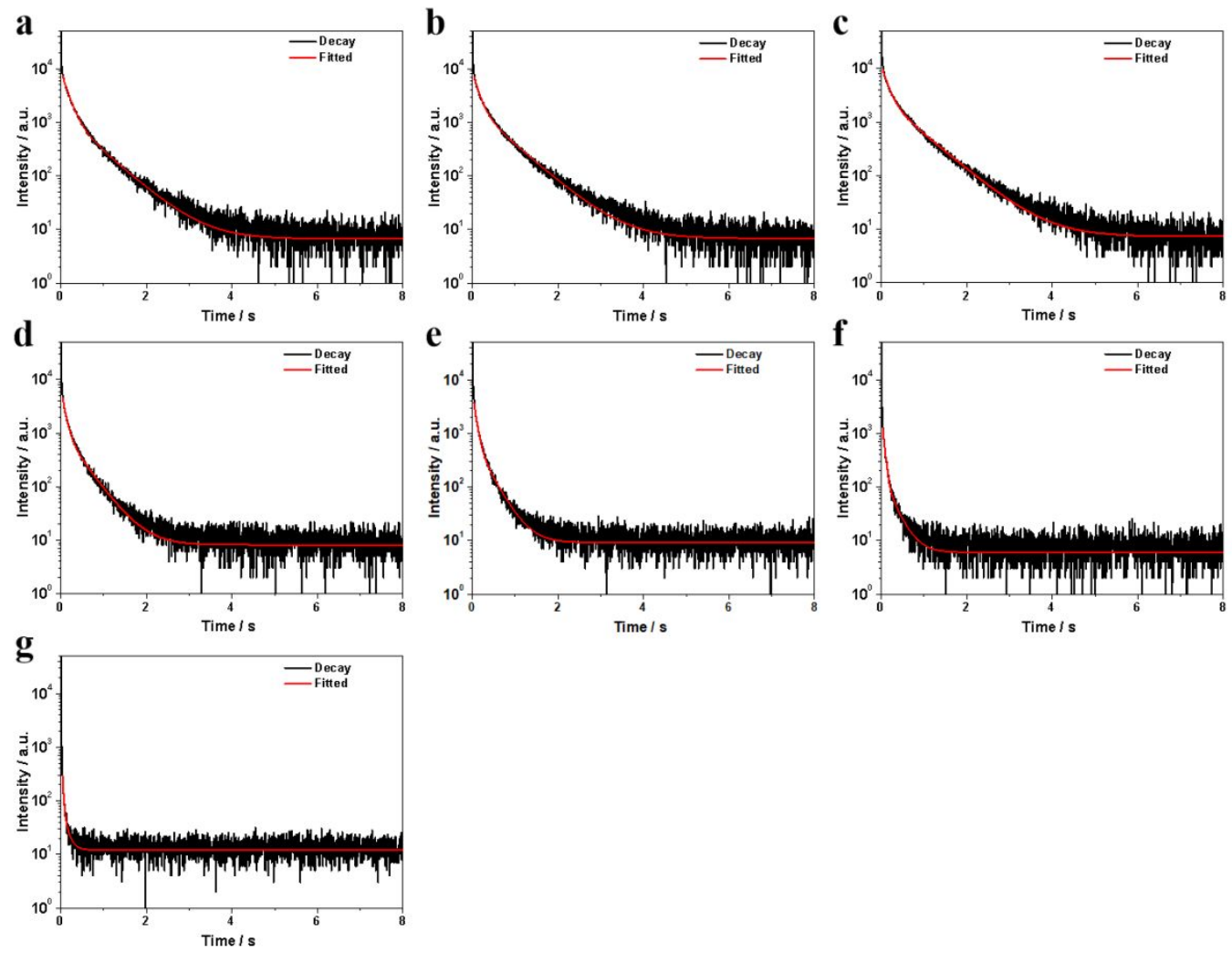

Figure S3. The fitting results of RTP decay. Phosphorescence decay spectra and fitting curve of AN-CPDs-150 (a), AN-CPDs-180 (b), AN-CPDs (c), AN-CPDs-230 (d), AN-CPDs-250 (e), AN-CPDs-280 (f) and AN-CPDs-300 (g) under ambient conditions.

Note: The RTP decay curve of CPDs at room temperature was fitted by the double-exponential equation:

$I=A+B 1 \exp \left(-t / \tau_{1}\right)+B 2 \exp \left(-t / \tau_{2}\right)$

where $\tau_{1}$ and $\tau_{2}$ are the two components of the afterglow lifetime, respectively. B1 and B2 are the corresponding fitting parameters. The average RTP lifetime can be further estimated by the following equation:

$\tau=\left(\mathrm{B} 1 \tau_{1}^{2}+\mathrm{B} 2 \tau_{2}^{2}\right) /\left(\mathrm{B} 1 \tau_{1}+\mathrm{B} 2 \tau_{2}\right)$ 


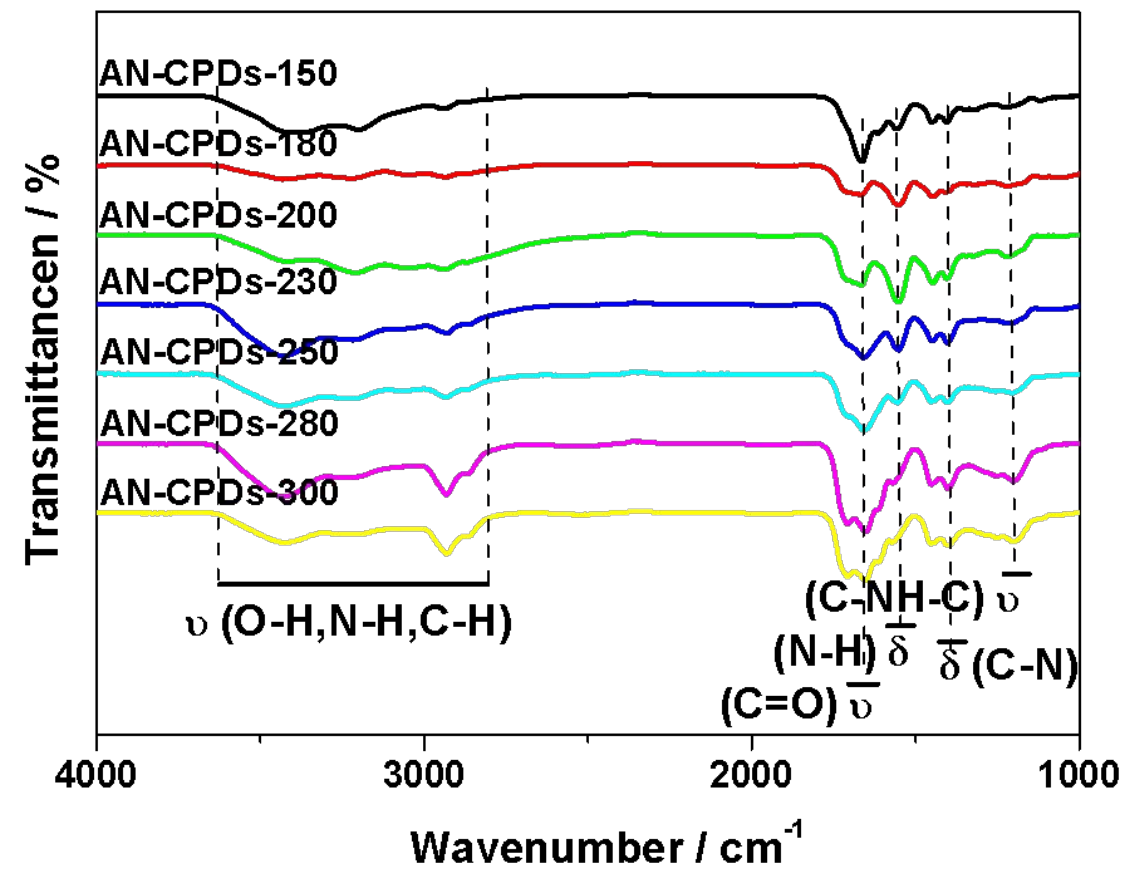

Figure S4. FT-IR spectra of the AN-CPDs with different carbonization degree. For AN-CPDs with different carbonization degree, they all show stretching vibrations of $\mathrm{O}-\mathrm{H}, \mathrm{N}-\mathrm{H}$ and $\mathrm{C}-\mathrm{H}$ exist broad absorption peaks from about $3650 \mathrm{~cm}-1$ to $2700 \mathrm{~cm}-1$. The peaks at $1670 \mathrm{~cm}-1$ and $1215 \mathrm{~cm}-1$ are attributed to the stretching vibrations of $\mathrm{C}=\mathrm{O}$ and $\mathrm{C}-\mathrm{NH}-\mathrm{C}$. The characteristic absorption of bending vibration of N-H and C-N are observed at $1553 \mathrm{~cm}-1$ and $1400 \mathrm{~cm}-1$, respectively. 

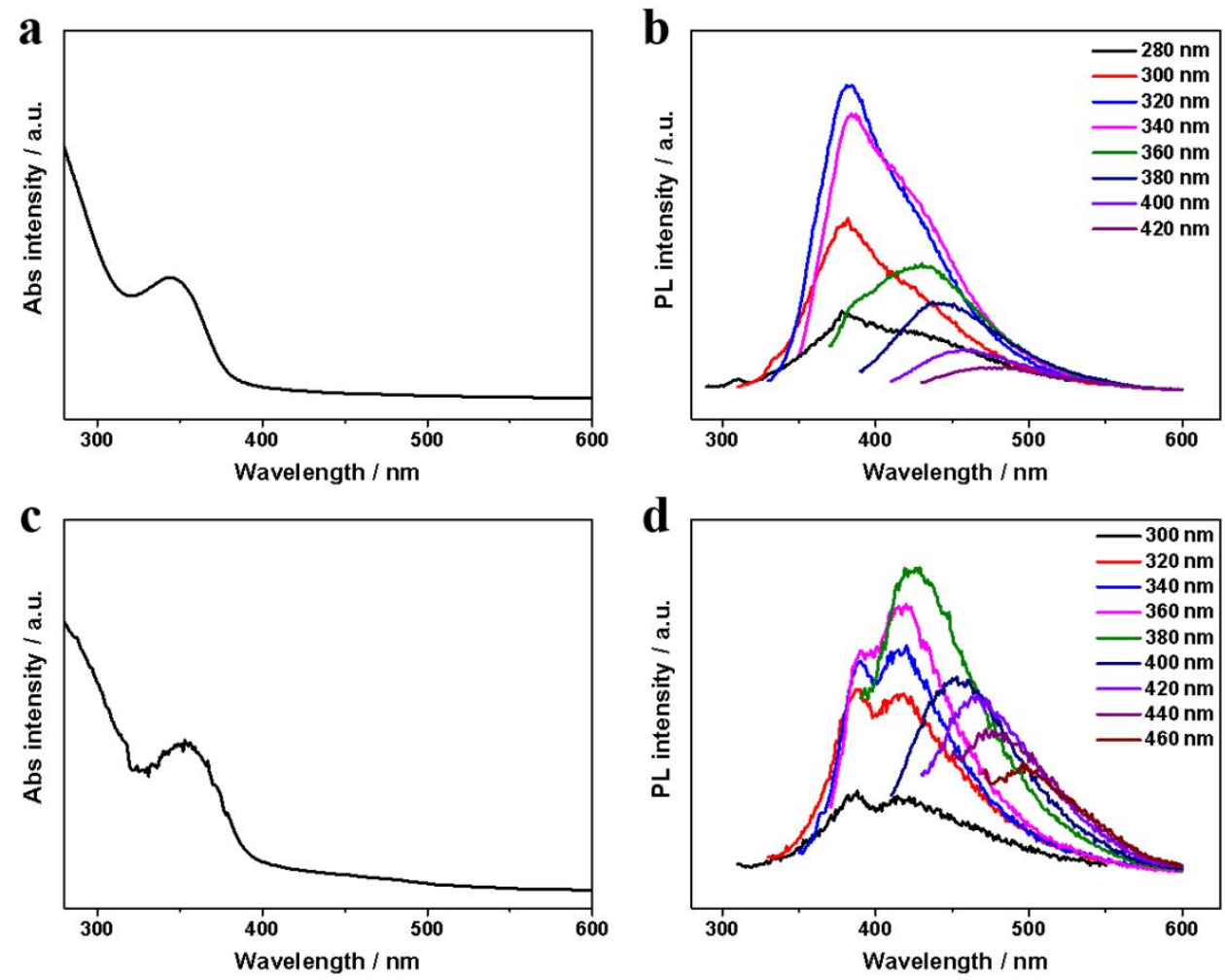

Figure S5. The optical properties of AN-CPDs-150. a, b) UV-Vis absorption spectrum and PL emission spectra under different excitation wavelengths of AN-CPDs-150 aqueous solution. c, d) UV-Vis absorption spectrum and PL emission spectra under different excitation wavelengths of AN-CPDs-150 solid. 

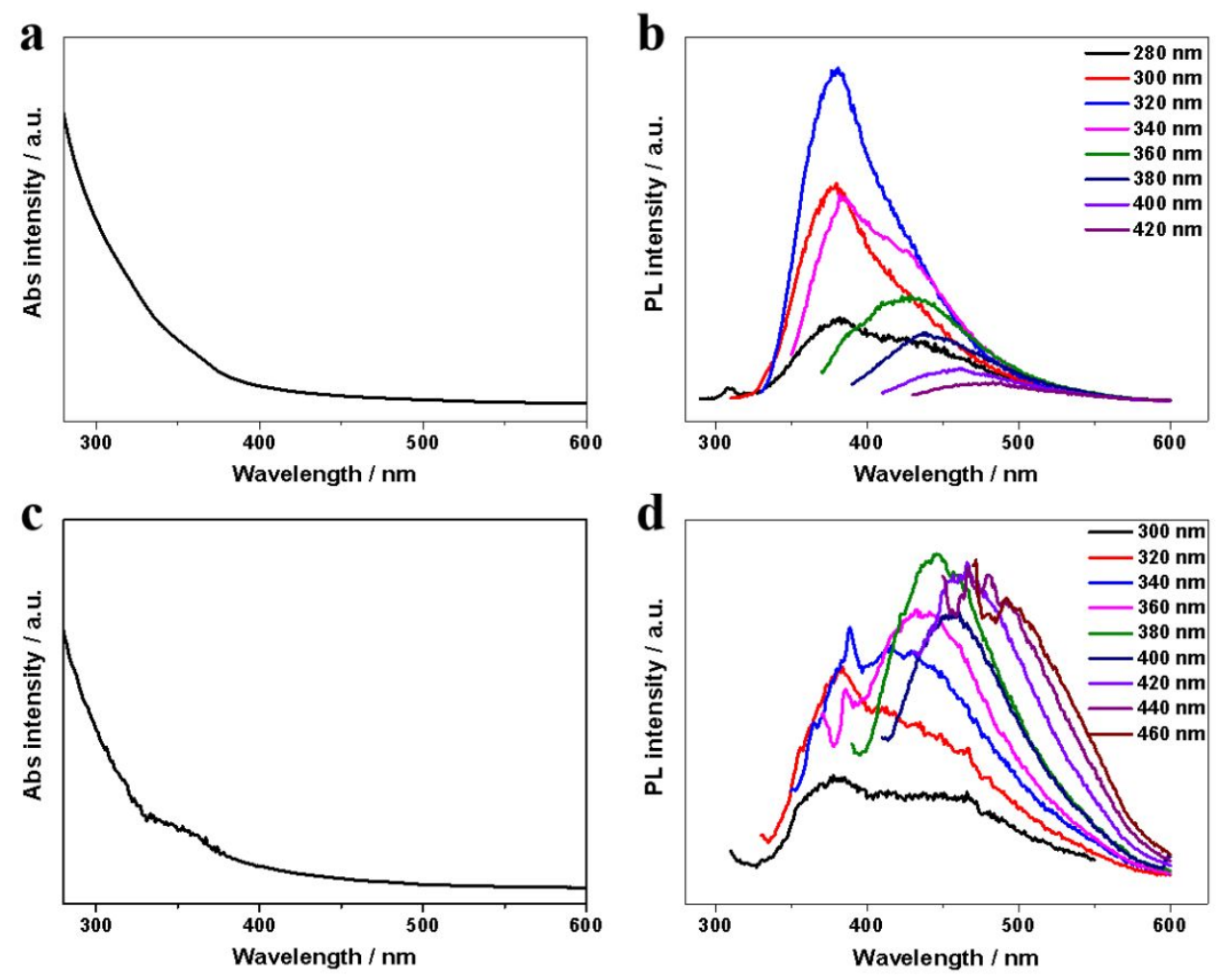

Figure S6. The optical properties of AN-CPDs-180. a, b) UV-Vis absorption spectrum and PL emission spectra under different excitation wavelengths of AN-CPDs-180 aqueous solution. c, d) UV-Vis absorption spectrum and PL emission spectra under different excitation wavelengths of AN-CPDs-180 solid. 

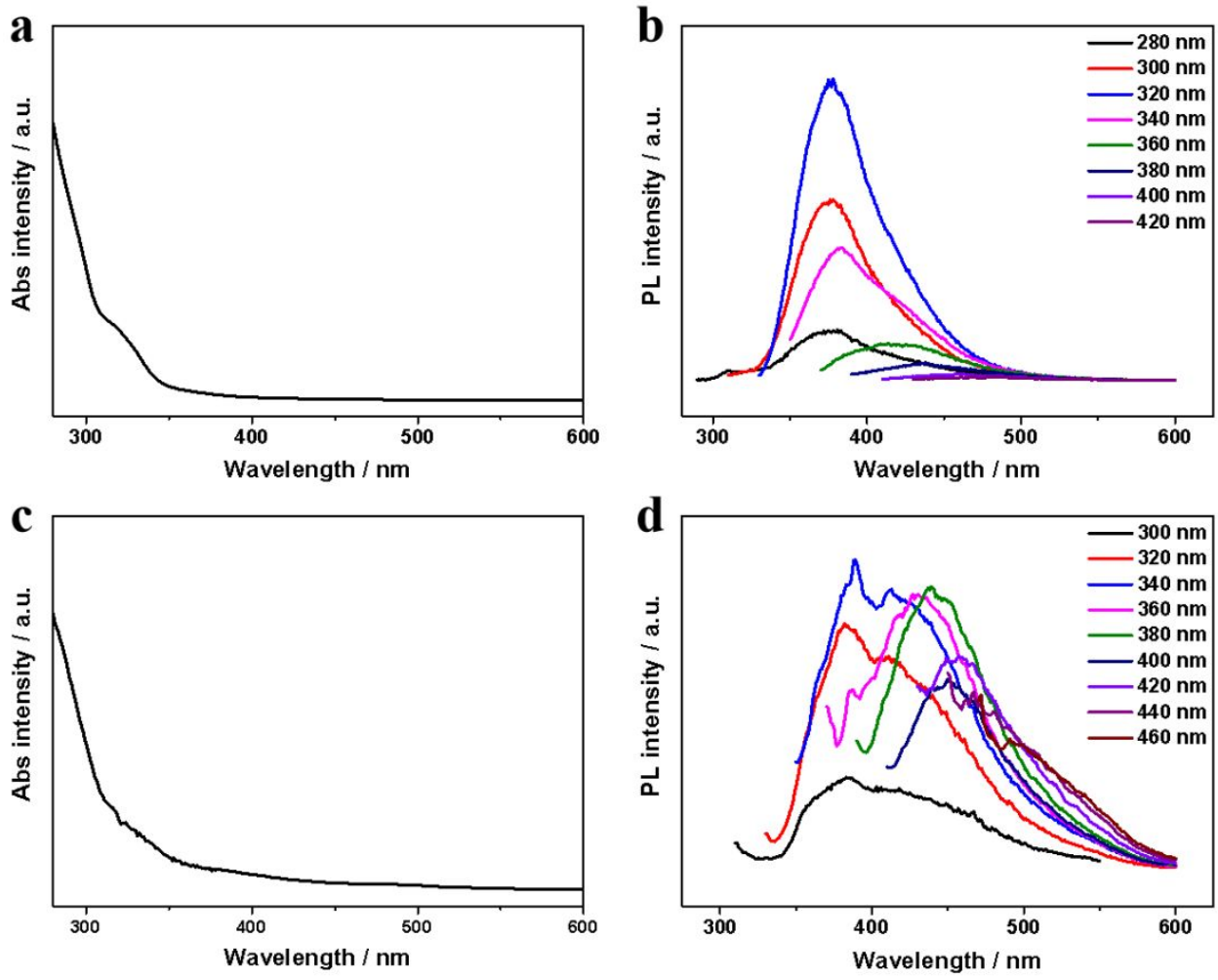

Figure S7. The optical properties of AN-CPDs-230. a, b) UV-Vis absorption spectrum and PL emission spectra under different excitation wavelengths of AN-CPDs-230 aqueous solution. c, d) UV-Vis absorption spectrum and PL emission spectra under different excitation wavelengths of AN-CPDs-230 solid. 

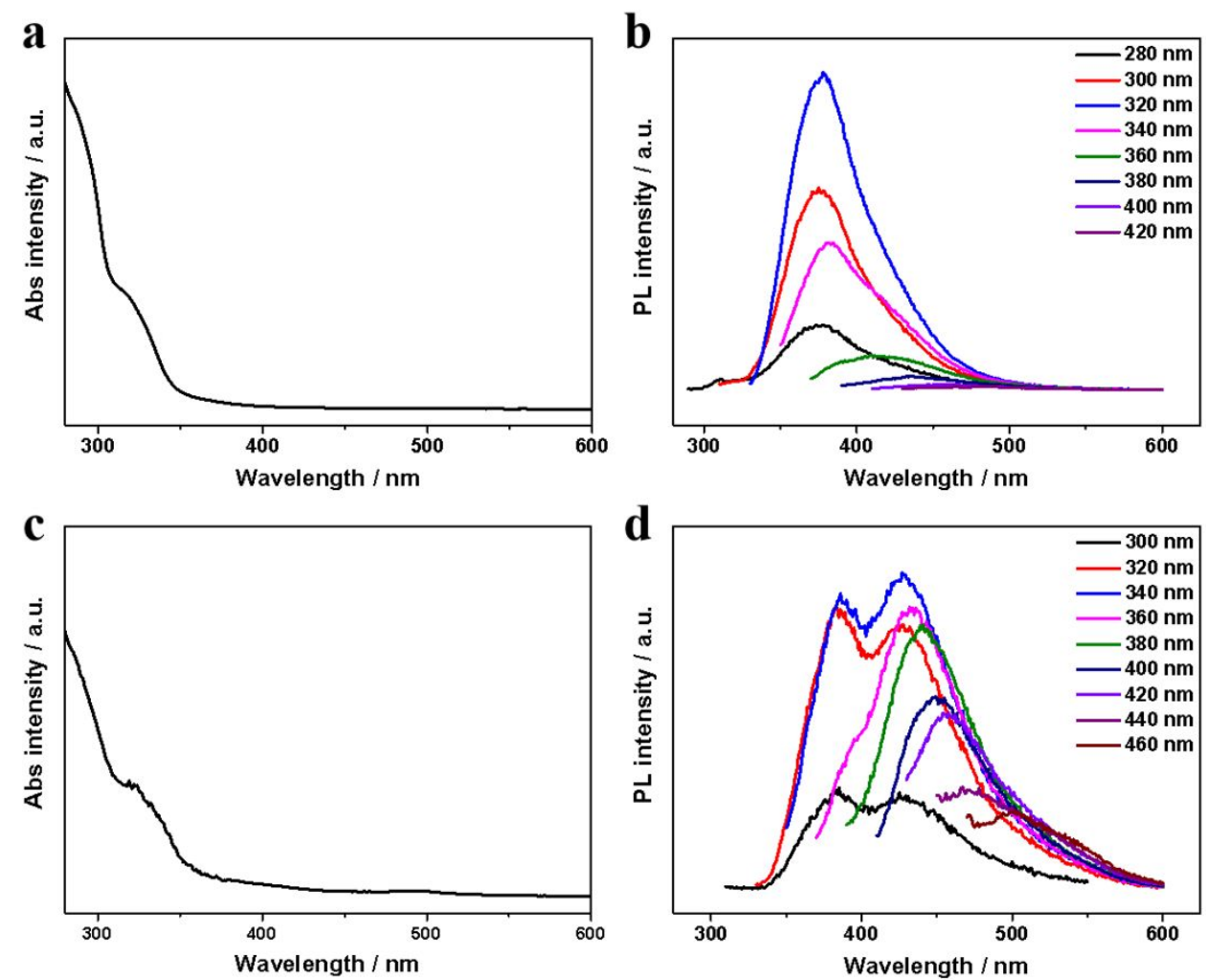

Figure S8. The optical properties of AN-CPDs-250. a, b) UV-Vis absorption spectrum and PL emission spectra under different excitation wavelengths of AN-CPDs-250 aqueous solution. c, d) UV-Vis absorption spectrum and PL emission spectra under different excitation wavelengths of AN-CPDs-250 solid. 

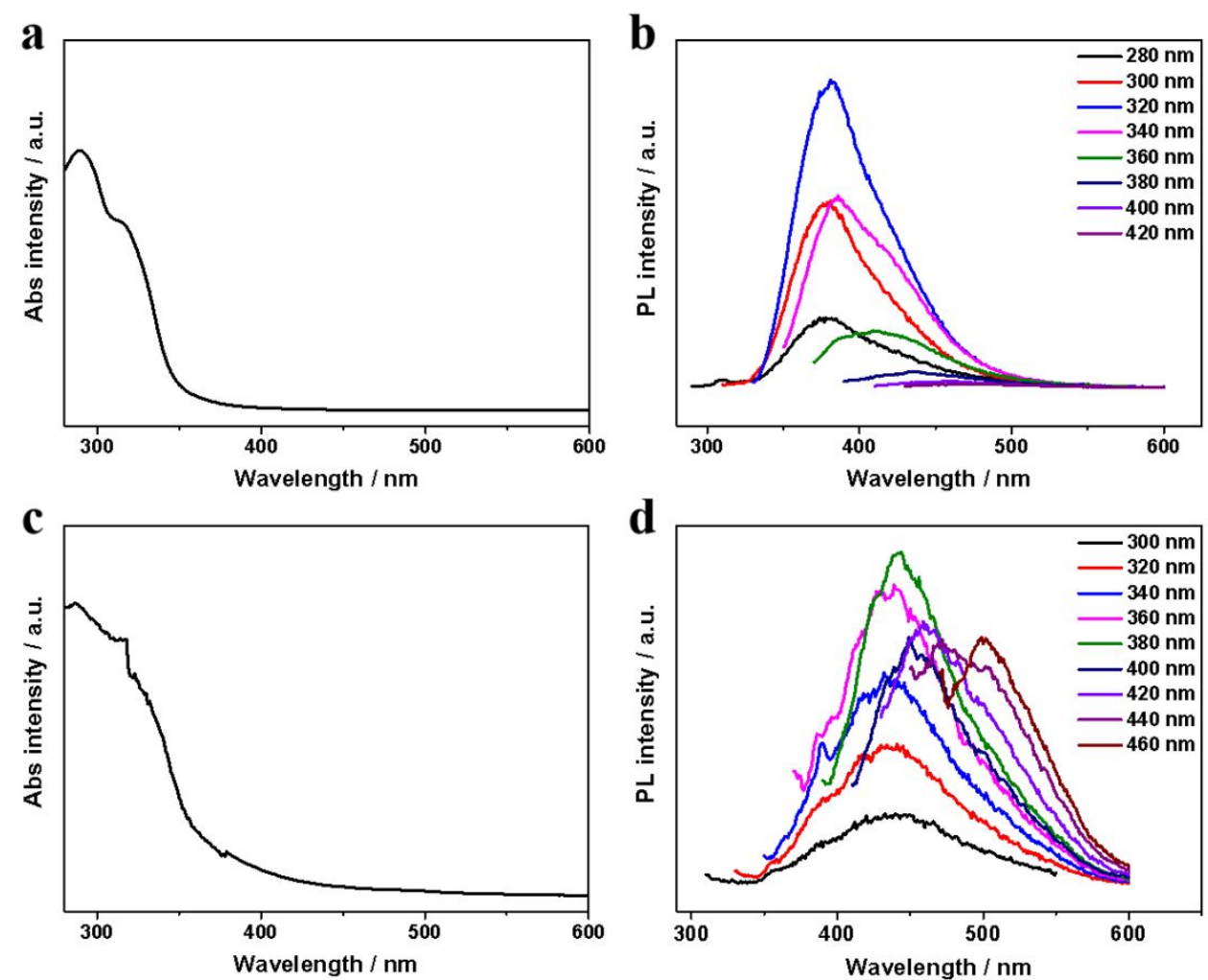

Figure S9. The optical properties of AN-CPDs-280. a, b) UV-Vis absorption spectrum and PL emission spectra under different excitation wavelengths of AN-CPDs-280 aqueous solution. c, d) UV-Vis absorption spectrum and PL emission spectra under different excitation wavelengths of AN-CPDs-280 solid. 

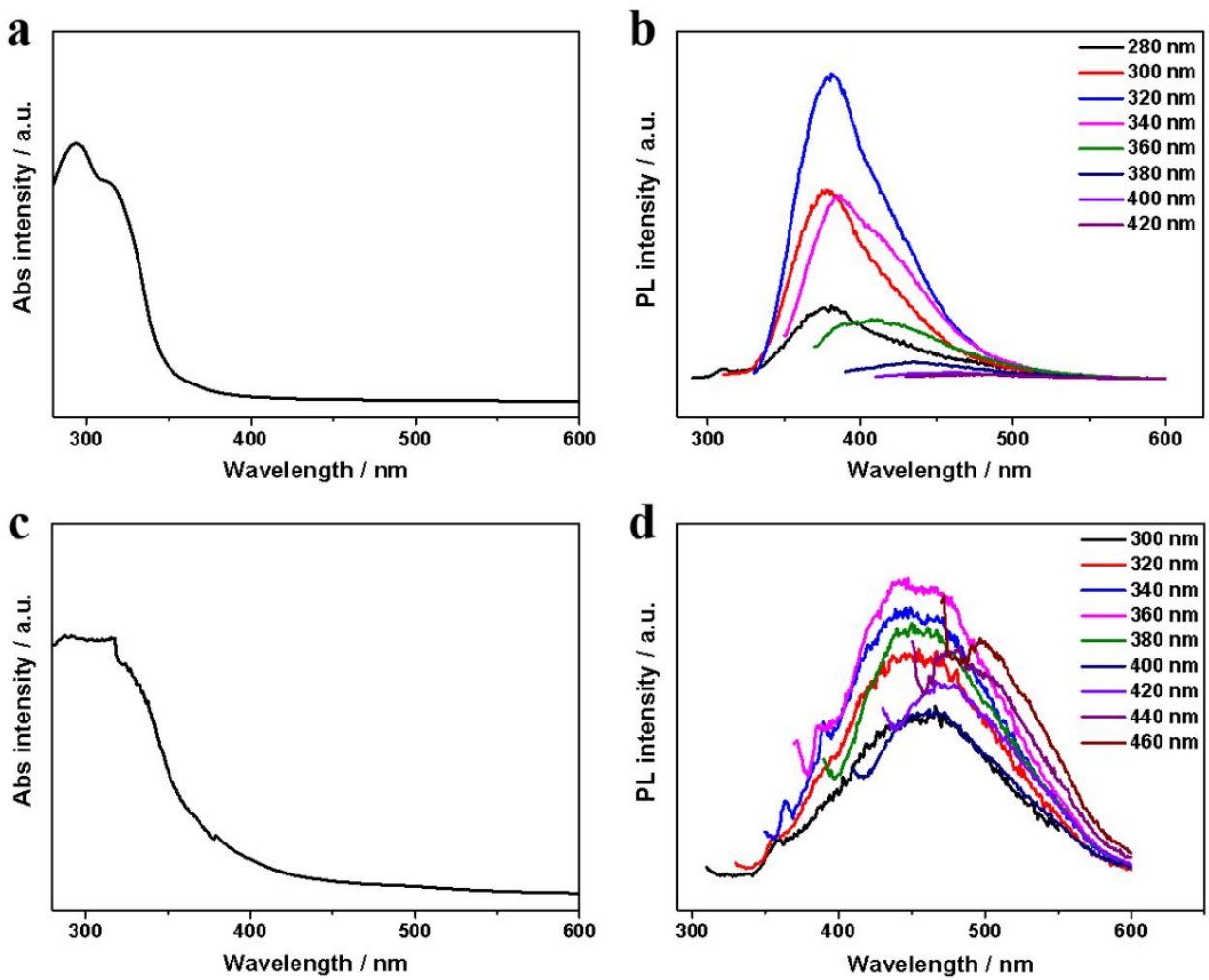

Figure S10. The optical properties of AN-CPDs-300. a, b) UV-Vis absorption spectrum and PL emission spectra under different excitation wavelengths of AN-CPDs-300 aqueous solution. c, d) UV-Vis absorption spectrum and PL emission spectra under different excitation wavelengths of AN-CPDs-300 solid.

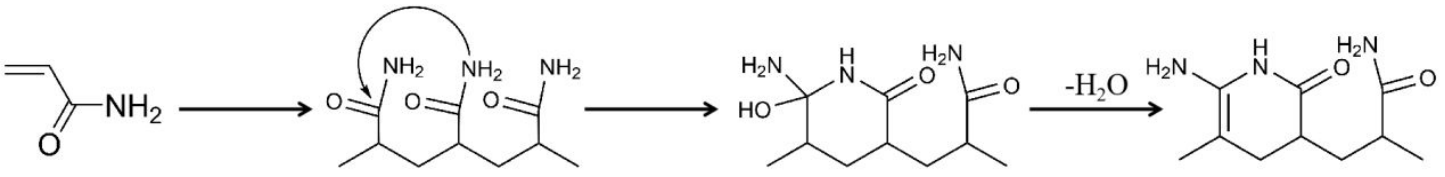

Figure S11. Scheme of possible structures formed during the hydrothermal treatment process. 


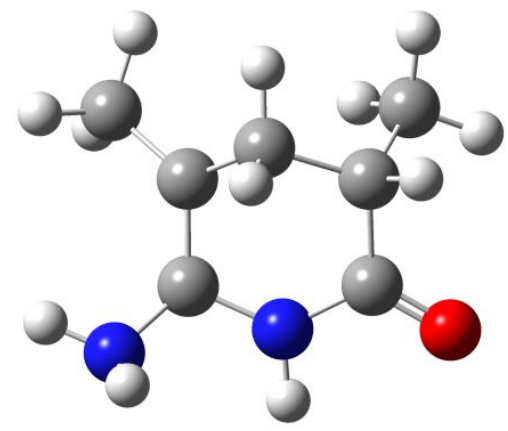

Figure S12. The optimized conformation of the predicted cyclic imide structure. 

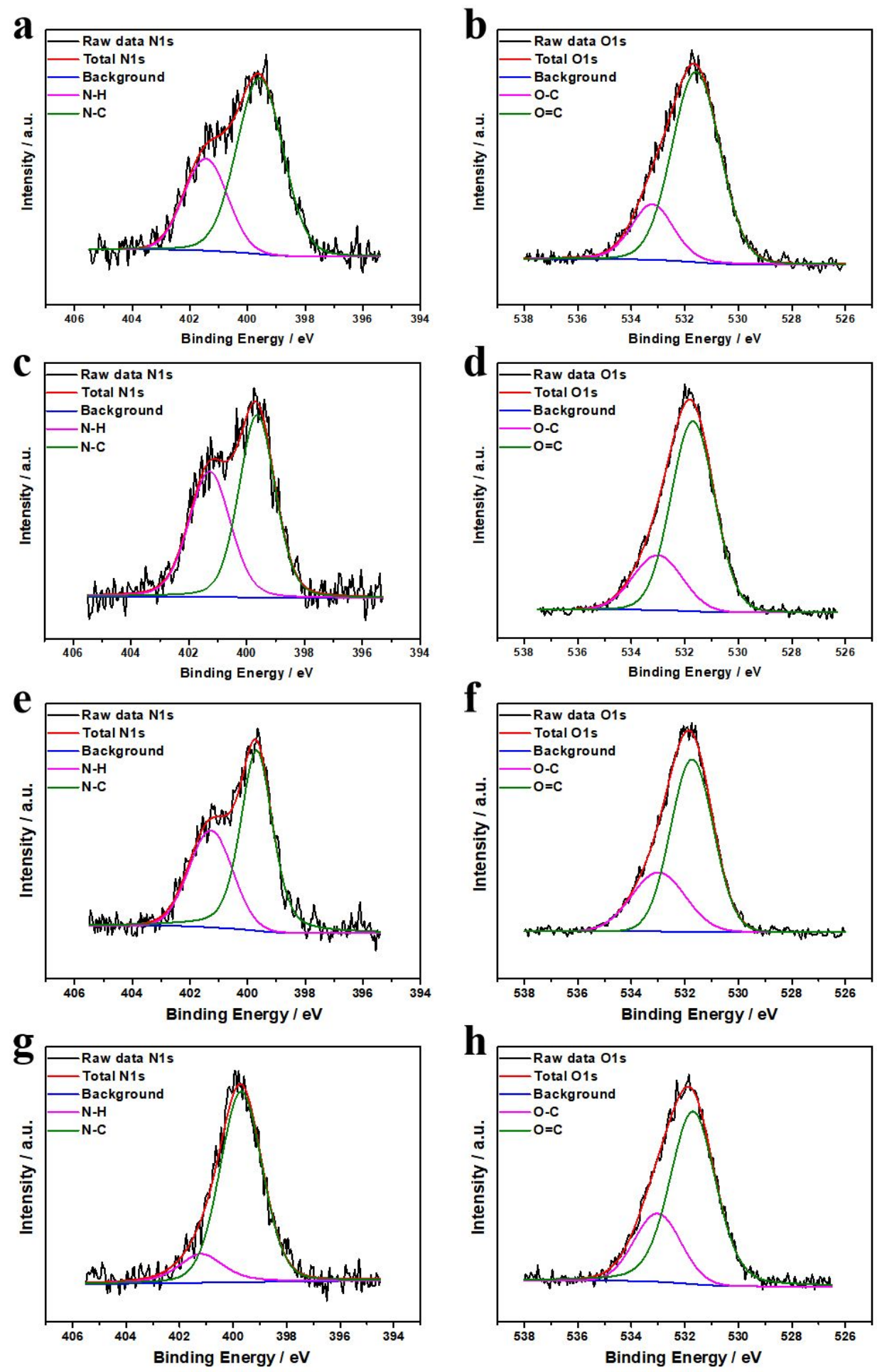

Figure S13. $N$ 1s and $O$ 1s high-resolution XPS spectra of the AN-CPDs-150 (a, b), AN-CPDs (c, d), AN-CPDs-250 (e, f) and AN-CPDs-300 (g, h). 


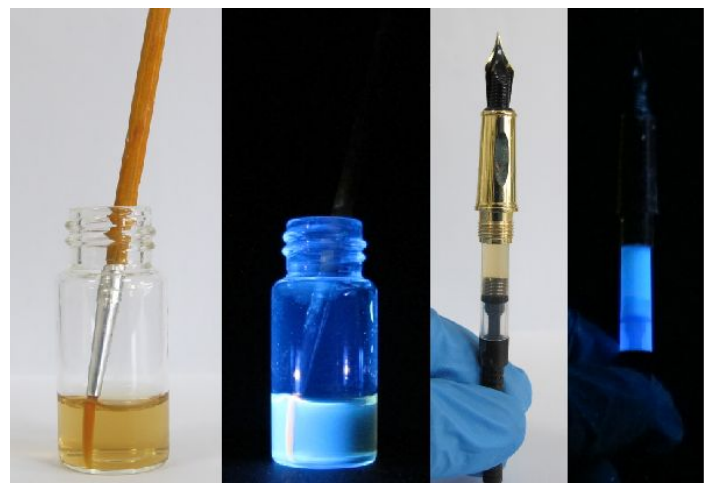

Figure S14. The photo display of AN-CPDs as the encrypted security ink.

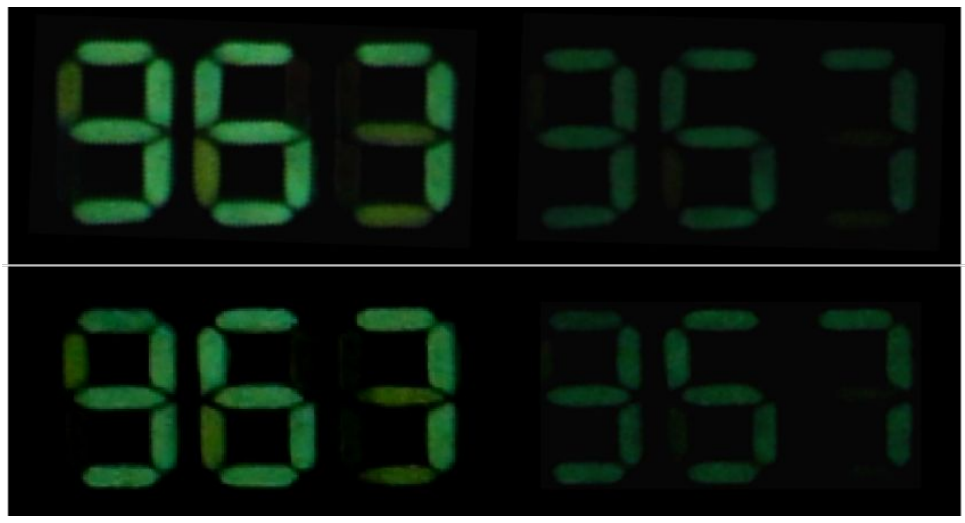

Figure S15. RTP emission performance contrast after 200 days (above: 200 days ago, below: 200 days later), which show the good stability of the RTP pattern. 
Table S1. The RTP lifetime fitting results of the AN-CPDs with different carbonization degree and CPDs-contrast.

\begin{tabular}{lccccc}
\hline \multicolumn{1}{c}{ Sample } & $\boldsymbol{\tau}_{\mathbf{1}}(\mathbf{m s})$ & $\mathbf{f}_{\mathbf{1}}(\mathbf{\%})$ & $\boldsymbol{\tau}_{\mathbf{2}}(\mathbf{m s})$ & $\mathbf{f}_{\mathbf{2}}(\mathbf{\%})$ & $\boldsymbol{\tau}_{\text {avg }}(\mathbf{m s})$ \\
\hline AN-CPDs-150 & 146.3 & 51.90 & 618.7 & 48.10 & 373.5 \\
AN-CPDs-180 & 129.8 & 38.59 & 628.6 & 61.41 & 436.1 \\
AN-CPDs & 133.7 & 35.61 & 650.6 & 64.39 & 466.5 \\
AN-CPDs-230 & 83.0 & 44.41 & 397.4 & 55.59 & 257.8 \\
AN-CPDs-250 & 66.5 & 52.78 & 295.8 & 47.22 & 174.8 \\
AN-CPDs-280 & 45.4 & 61.95 & 234.0 & 38.05 & 117.2 \\
AN-CPDs-300 & 22.9 & 50.58 & 100.8 & 49.42 & 61.4 \\
CPDs-contrast & 46.3 & 42.81 & 203.6 & 57.19 & 136.3 \\
\hline
\end{tabular}

Table S2. The theoretical calculations results using the delta-SCF method.

\begin{tabular}{ccc}
\hline & $\mathbf{S}_{\mathbf{0}}$ & $\mathbf{T}_{\mathbf{1}}$ \\
\hline Energy(Hartree) & -458.72578071 & -458.63106727 \\
\hline
\end{tabular}

Note: $\mathrm{T}_{1}-\mathrm{S}_{0}=0.09471344$ Hartree $=(0.09471344 * 27.2114) \mathrm{eV}=2.56 \mathrm{eV}$

Table S3. Relative contents of different functional groups in the AN-CPDs-150, AN-CPDs, AN-CPDs-250, AN-CPDs-300, based on the analysis of C1s high-resolution XPS spectra.

\begin{tabular}{lccc}
\hline \multicolumn{1}{c}{ sample } & $\mathbf{C}=\mathbf{O} / \mathbf{C}=\mathbf{N}$ & $\mathbf{C}-\mathbf{N} / \mathbf{C}-\mathbf{O}$ & $\mathbf{C}=\mathbf{C} / \mathbf{C}-\mathbf{C}$ \\
\hline AN-CPDs-150 & 288.367 & 285.823 & 284.567 \\
& $14.59 \%$ & $15.42 \%$ & $69.99 \%$ \\
AN-CPDs & 288.321 & 285.588 & 284.586 \\
& $12.32 \%$ & $11.42 \%$ & $76.26 \%$ \\
AN-CPDs-250 & 288.343 & 285.847 & 284.726 \\
& $8.75 \%$ & $10.31 \%$ & $80.94 \%$ \\
AN-CPDs-300 & 288.33 & 285.764 & 284.612 \\
& $6.95 \%$ & $9.72 \%$ & $83.33 \%$ \\
\hline
\end{tabular}

